\title{
Strange baryons with two heavy quarks
}

\author{
Marek Karliner ${ }^{1, *}$ and Jonathan L. Rosner ${ }^{2, \dagger}$ \\ ${ }^{1}$ School of Physics and Astronomy Raymond and Beverly Sackler Faculty \\ of Exact Sciences Tel Aviv University, Tel Aviv 69978, Israel \\ ${ }^{2}$ Enrico Fermi Institute and Department of Physics University of Chicago, \\ 5620 S. Ellis Avenue, Chicago, Illinois 60637, USA
}

(Received 14 March 2018; published 10 May 2018)

\begin{abstract}
The LHCb Experiment at CERN has observed a doubly-charmed baryon $\Xi_{c c}^{++}=c c u$ with a mass of $3621.40 \pm 0.78 \mathrm{MeV}$, consistent with many predictions. We use the same methods that led us to predict $M\left(\Xi_{c c}, J^{P}=1 / 2^{+}\right)=3627 \pm 12 \mathrm{MeV}$ and $M\left(\Xi_{c c}^{*}, J^{P}=3 / 2^{+}\right)=3690 \pm 12 \mathrm{MeV}$ to predict $M\left(\Omega_{c c}^{+}, J^{P}=1 / 2^{+}\right)=3692 \pm 16 \mathrm{MeV}$ and $M\left(\Omega_{c c}^{*}, J^{P}=3 / 2^{+}\right)=3756 \pm 16 \mathrm{MeV}$. Production and decay are discussed briefly, and predictions for $M\left(\Omega_{b c}\right)$ and $M\left(\Omega_{b b}\right)$ are included.
\end{abstract}

DOI: $10.1103 /$ PhysRevD.97.094006

\section{INTRODUCTION}

The LHCb Experiment at CERN has observed a doublycharmed baryon $\Xi_{c c}^{++}=c c u$ with a mass of $3621.40 \pm$ $0.78 \mathrm{MeV}$ [1]. This value is consistent with several predictions, including our value of $3627 \pm 12 \mathrm{MeV}[2,3]$. It is more than $100 \mathrm{MeV}$ above a candidate $\Xi_{c c}^{+}$for an isospin partner claimed by the SELEX Collaboration [4], but not seen by others. Here we use similar methods to those in Ref. [2] and earlier works [5] to predict the mass of the ground-state $c c s$ state with spin-parity $J^{P}=1 / 2^{+}$ $M\left(\Omega_{c c}^{+}\right)=3692 \pm 16 \mathrm{MeV}$ and its hyperfine partner with $J^{P}=3 / 2^{+}, M\left(\Omega_{c c}^{*}\right)=3756 \pm 16 \mathrm{MeV}$. Binding effects lead the difference between the strange and nonstrange doubly charmed baryon masses to be less than half the constituent-quark mass difference between the strange and nonstrange light quarks. These results were obtained using constituent-quark masses appropriate for baryons. Use of quark masses universal for baryons and mesons leads to $M\left(\Omega_{c c}\right)$ and $M\left(\Omega_{c c}^{*}\right)$ about $40 \mathrm{MeV}$ higher, with similar systematic variations expected for $\Omega_{b c}$ and $\Omega_{b b}$, due mostly to uncertainty in how strongly a strange quark binds to a heavy diquark.

In Sec. II we list contributions to $M\left(\Omega_{c c}^{+}\right)$that are straightforward extrapolations of the calculation of $M\left(\Xi_{c c}\right)$. The pair of charmed quarks is treated as a $(c c)$ diquark antisymmetric (a $\left.3^{*}\right)$ in color and hence symmetric

\footnotetext{
*marek@proton.tau.ac.il

rosner@hep.uchicago.edu
}

Published by the American Physical Society under the terms of the Creative Commons Attribution 4.0 International license. Further distribution of this work must maintain attribution to the author(s) and the published article's title, journal citation, and DOI. Funded by SCOAP . in spin $(S=1)$. The difference in binding between a $(c c)$ diquark and a strange quark in comparison with binding between $(c c)$ and a nonstrange quark is discussed in Sec. III. Results using quark masses appropriate for both mesons and baryons are treated in Sec. IV. Production and decay are treated briefly in Sec. V, predictions for $M\left(\Omega_{b c}\right)$ and $M\left(\Omega_{b b}\right)$ are presented in Sec. VI, while results and comments on other work are collected in Sec. VII.

\section{EXTRAPOLATIONS FROM $\Xi_{c c}$ PREDICTION}

We compare the contributions to the $\Xi_{c c}$ mass studied in Ref. [2] to similar contributions to the $\Omega_{c c}$ mass in Table I. We take quarks in a baryon to have effective masses $m_{q}^{b}=363 \mathrm{MeV}(q=u$ or $d), m_{s}^{b}=538 \mathrm{MeV}$, $m_{c}^{b}=1710.5 \mathrm{MeV}$, and $m_{b}^{b}=5043.5 \mathrm{MeV}$. We ignore isospin splitting, treated in [6] and references therein.

The effect of the spin-spin interaction between the $q$ quark and the $(c c)$ diquark is parametrized by a term $-4 a / m_{q}^{b} m_{c}^{b}$, while that between $s$ and $(c c)$ is parametrized by $-4 a^{\prime} / m_{s}^{b} m_{c}^{b}$ with $a^{\prime}=a m_{s}^{b} / m_{q}^{b}$ taken so that the two terms

TABLE I. Comparison of contributions to the mass of the lightest doubly charmed baryon $\Xi_{c c}$ [2] with corresponding contributions to the mass of $\Omega_{c c}$.

\begin{tabular}{lcccc}
\hline \hline \multicolumn{2}{c}{$\Xi_{c c}=c c q$} & & \multicolumn{2}{c}{$\Omega_{c c}=c c s$} \\
\cline { 1 - 2 } \cline { 5 - 5 } Contribution & Value $(\mathrm{MeV})$ & & Contribution & Value $(\mathrm{MeV})$ \\
\hline $2 m_{c}^{b}+m_{q}^{b}$ & 3789.0 & & $2 m_{c}^{b}+m_{s}^{b}$ & 3959.0 \\
$c c$ binding & -129.0 & & $c c$ binding & -129.0 \\
$a_{c c} /\left(m_{c}^{b}\right)^{2}$ & 14.2 & & $a_{c c} /\left(m_{c}^{b}\right)^{2}$ & 14.2 \\
$-4 a / m_{q}^{b} m_{c}^{b}$ & -42.4 & & $-4 a^{\prime} / m_{s}^{b} m_{c}^{b}$ & -42.4 \\
Total & $3626.8 \pm 12$ & & Subtotal & $3801.8 \pm 12$ \\
\hline \hline
\end{tabular}


have the same strength. This is motivated by comparing the spin-spin interaction in the $c \bar{s}$ and $c \bar{q}$ systems: $M\left(D_{s}^{*}\right)-$ $M\left(D_{s}\right)=143.8 \mathrm{MeV}$ is almost the same as $M\left(D^{*}\right)-$ $M(D)=141.4 \mathrm{MeV}$. The smaller magnetic moment of $s$ is compensated by a larger wave function at the origin in the $c \bar{s}$ system. We assume a similar compensation is taking place here. For the mass of the $\Omega_{c c}^{*}\left(J^{P}=3 / 2^{+}\right)$, we replace the term $-4 a^{\prime} / m_{s}^{b} m_{c}^{b}=-42.4 \mathrm{MeV}$ by $+2 a^{\prime} / m_{s}^{b} m_{c}^{b}=+21.2 \mathrm{MeV}, \quad$ so $\quad M\left[\Omega_{c c}^{*}\left(J^{P}=3 / 2^{+}\right)\right]-$ $M\left[\Omega_{c c}\left(J^{P}=1 / 2^{+}\right)\right]=63.6 \mathrm{MeV}$.

Our calculations of the masses of light hadrons, based on the ideas of Ref. [7], use constituent-quark masses and do not require separate binding energies. However, for systems without $q$ involving heavy quarks one must take into account additional binding. For example, when calculating the mass of the $S$-wave $c \bar{s}$ system, it was found necessary to include a supplemental binding energy of $69.9 \mathrm{MeV}$, while a binding energy of $258 \mathrm{MeV}$ was needed to describe $\mathrm{S}$-wave charmonium [2]. Hence the last energy in Table I represents a subtotal; we estimate the binding energy of $s$ with the diquark $(c c)$ in the next section.

\section{DIQUARK-LIGHT QUARK BINDING}

We shall interpolate between the $\bar{c} s$ and $\bar{c} c$ binding energies to find that between $(c c)$ and $s$. All three cases involve the interaction of a color antitriplet with a color triplet. We compare the masses $m_{1}, m_{2}$ of the constituents and reduced mass $\mu \equiv m_{1} m_{2} /\left(m_{1}+m_{2}\right)$ of the composite system in Table II. When discussing mesons, we use effective masses $m_{q}^{m}=310 \mathrm{MeV}, m_{s}^{m}=483 \mathrm{MeV}, m_{c}^{m}=$ $1663.3 \mathrm{MeV}$ and $m_{b}^{m}=5003.8 \mathrm{MeV}$ [2]. The mass of the $c c$ diquark is calculated to be $2 m_{c}^{b}-B(c c)+a_{c c} /\left(m_{c}^{b}\right)^{2}=$ $3421.0-129.0+14.2=3306.2 \mathrm{MeV}$. For use in subsequent discussion of the masses of $\Omega_{b c} \equiv b c s$ and $\Omega_{b b} \equiv b s s$, we include the binding energies between the diquark $(b c)$ and $s$ and between the diquark $(b b)$ and $s$. The mass of the $b c$ diquark is calculated to be $m_{b}^{b}+m_{c}^{b}-$ $B(b c)=5043.5+1710.5-(167.6 \pm 3)=6586.4 \pm 3 \mathrm{MeV}$,

TABLE II. Comparison of constituent masses and reduced masses in $\mathrm{MeV}$ for some systems of strange and $c$ or $b$ quarks, in a scheme with separate quark masses for mesons and baryons. Binding energies in $\mathrm{MeV}$ are also shown, with two different values averaged and errors reflecting half their difference for the $(c c) s,(b c) s$, and $(b b) s$ systems (see text).

\begin{tabular}{lcccc}
\hline \hline System & $m_{1}$ & $m_{2}$ & $\mu$ & $B$ \\
\hline $\bar{c} s$ & 1663.3 & 483 & 374.3 & 69.9 \\
$\bar{c} c$ & 1663.3 & 1663.3 & 831.6 & 258 \\
$(c c) s$ & 3306.2 & 538 & 462.7 & $109.4 \pm 10.5$ \\
$(b c) s$ & $6586.4^{\mathrm{a}}$ & 538 & 497.4 & $124.1 \pm 12.8$ \\
$(b b) s$ & 9813.4 & 538 & 510.0 & $129.4 \pm 13.4$ \\
\hline \hline
\end{tabular}

${ }^{a}$ Mass eigenstates of indefinite $b c$ spin; small hyperfine terms ignored. where the error reflects uncertainty in the $b c$ binding energy. As the mass eigenstates are of indefinite $b c$ spin (rather, they are approximately states of definite $c s$ spin), we ignore small hyperfine effects. The mass of the $b b$ diquark is calculated to be $2 m_{b}^{b}-B(b b)+a_{b b} /\left(m_{b}^{b}\right)^{2}=$ $10087.0-281.4+7.8=9813.4 \mathrm{MeV}$.

The reduced mass of the $(c c) s$ system lies between those of $\bar{c} s$ and $\bar{c} c$. Assuming a power-law dependence on $\mu$, $B=A \mu^{p}$, gives $p=1.636$ and $B((c c) s)=98.9 \mathrm{MeV}$. An alternate method makes use of the Feynman-Hellmann theorem [8], which relates the derivative of an energy expectation value with respect to a parameter $\mu$ to the expectation value of the derivative of the Hamiltonian:

$$
\frac{d E_{\mu}}{d \mu}=\left\langle\frac{d H_{\mu}}{d \mu}\right\rangle
$$

In the present case, the right-hand side is $-(1 / \mu)\langle T\rangle$, where $T$ is the kinetic energy. Let us now assume $\langle T\rangle$ is independent of the reduced mass. This is indeed the case for a logarithmic potential $[9,10]$, which has been shown to suitably interpolate between charmonium and bottomonium. We shall assume $T$ is constant also for our interpolation. Then the shift in binding energy between a system with reduced mass $\mu_{1}$ and one with $\mu_{2}$ is

$$
\Delta B=\langle T\rangle \int_{\mu_{1}}^{\mu_{2}} \frac{d \mu}{\mu}=\langle T\rangle \ln \frac{\mu_{2}}{\mu_{1}} .
$$

The binding energy increases with increased reduced mass, as expected. One can determine $\langle T\rangle=235.6 \mathrm{MeV}$ by comparing $\bar{c} s$ and $\bar{c} c$ binding energies, yielding

$$
\begin{aligned}
B((c c) s) & =B(\bar{c} s)+\langle T\rangle \ln \frac{462.7}{374.3} \\
& =69.9+50=119.9 \mathrm{MeV} .
\end{aligned}
$$

The average of the two determinations is $109.4 \pm 10.5 \mathrm{MeV}$, where we take the error to be half of their difference. Similar methods apply to the estimates of $B((b c) s)$ and $B((b b) s)$ quoted in Table II, where the averages are those of the power-law (lesser value) and Feynman-Hellmann (greater value) methods of interpolation. Subtracting this from the subtotal in Table I, whose error was assumed to be the same as in the calculations of $M\left(\Xi_{c c}\right)$, and adding the error of $\pm 10.5 \mathrm{MeV}$ in quadrature, we find $M\left(\Omega_{c c}\right)=3692 \pm 16 \mathrm{MeV}, M\left(\Omega_{c c}^{*}\right)=3756 \pm 16 \mathrm{MeV}$.

\section{UNIVERSAL QUARK MASSES}

For many years it has been realized that fits to baryon masses require constituent quarks about $55 \mathrm{MeV}$ heavier than those in fits to low-lying mesons $[11,12]$. An alternative, secondary, description [13] makes use of quark masses appropriate for both mesons and baryons, adding a term $S=165.1 \mathrm{MeV}$ to characterize the extra mass in a 
TABLE III. Contributions to $M\left(\Omega_{c c}\right)$ and $M\left(\Xi_{c c}\right)$ in a picture with identical quark masses for mesons and baryons. $a_{\text {uqm }}$ and $a_{u q m}^{\prime}$ denote the strengths of $c q$ and $c s$ color hyperfine coupling appropriate for universal quark masses [13].

\begin{tabular}{lcccc}
\hline \hline \multicolumn{2}{c}{$M\left(\Omega_{c c}\right)$} & & \multicolumn{2}{c}{$M\left(\Xi_{c c}\right)$} \\
\cline { 1 - 2 } \cline { 5 - 6 } Contribution & Value $(\mathrm{MeV})$ & & Contribution & Value $(\mathrm{MeV})$ \\
\hline $2 m_{c}+m_{s}$ & 3793.4 & & $2 m_{c}+m_{q}$ & 3619.7 \\
$c c$ binding & -121.3 & & $c c$ binding & -121.3 \\
$S$ & 165.1 & & $S$ & 165.1 \\
$a_{c c} /\left(m_{c}\right)^{2}$ & 14.2 & & $a_{c c} /\left(m_{c}\right)^{2}$ & 14.2 \\
$-4 a_{\text {uqm }}^{\prime} / m_{s} m_{c}$ & -37.6 & & $-4 a_{\text {uqm }} / m_{q} m_{c}$ & -37.6 \\
Subtotal & $3813.8 \pm 12$ & & Total & $3640.1 \pm 12$ \\
\hline \hline
\end{tabular}

baryon due to a string junction [14]. The contributions to $M\left(\Omega_{c c}\right)$, before accounting of binding between the $(c c)$ diquark and the strange quark, are shown in Table III. Also shown are contributions to $M\left(\Xi_{c c}\right)$ in this scheme. Here $m_{q}=308.5 \mathrm{MeV}, m_{s}=482.2 \mathrm{MeV}, m_{c}=1655.6 \mathrm{MeV}$, and $m_{b}=4988.6 \mathrm{MeV}$ [13].

The $(c c)$ diquark's mass is $M\left(c c, 3^{*}\right)=2(1655,6)-$ $121.3+14.2=3204.1 \mathrm{MeV}$. To account for binding between the $s$ quark and the $(c c)$ diquark, we interpolate as before, with the results shown in Table IV. The binding energy in the $\bar{c} s$ system has been calculated as $B(\bar{c} s)=-\left[3 M\left(D_{s}^{*}\right)+M\left(D_{s}\right)\right] / 4+m_{s}+m_{c}=-[3(2112.1)+$ $1968.3] / 4+482.2+1655.6 \mathrm{MeV}=61.65 \mathrm{MeV}$.

Interpolating via a power law with $B=A \mu^{p}$ one finds $p=1.721, \mathrm{~B}((\mathrm{cc}) \mathrm{s})=75.2 \mathrm{MeV}$, while interpolating via the Feynman-Hellmann theorem (2) one finds $\langle T\rangle=$ 227.4 MeV and $B((c c) s)=87.9 \mathrm{MeV}$. Hence $B((c c) s)=$ $81.6 \pm 6.4 \mathrm{MeV}, \quad$ implying $\quad M\left(\operatorname{ccs}, 1 / 2^{+}\right)=3732 \pm$ $14 \mathrm{MeV}$. This is $40 \mathrm{MeV}$ above the value we obtained with separate quark masses for meson and baryons. The uncertainty reflects in part the uncertainty in estimating the binding energy between a strange quark and the heavy

TABLE IV. Constituent and reduced masses in $\mathrm{MeV}$ for interpolation to find binding energy between $s$ and heavy diquarks $(c c),(b c)$, and $(b b)$, in a scheme with common quark masses for mesons and baryons. For the heavy diquark systems two different values have been averaged; errors reflect half their difference.

\begin{tabular}{lcrcc}
\hline \hline System & $m_{1}$ & $m_{2}$ & $\mu$ & $B$ \\
\hline $\bar{c} s$ & 1655.6 & 482.2 & 373.4 & 61.65 \\
$\bar{c} c$ & 1655.6 & 1655.6 & 827.8 & $242.7^{\mathrm{a}}$ \\
$(c c) s$ & 3204.1 & 482.2 & 419.1 & $81.6 \pm 6.4$ \\
$(b c) s$ & $6484.9^{\mathrm{b}}$ & 482.2 & 448.8 & $94.0 \pm 9.4$ \\
$(b b) s$ & 9718.9 & 482.2 & 459.4 & $98.4 \pm 10.4$ \\
\hline \hline
\end{tabular}

\footnotetext{
${ }^{\mathrm{a}}$ From Ref. [13].

${ }^{b}$ Mass eigenstates of indefinite $b c$ spin; small hyperfine terms ignored.
}

diquark. A precise measurement of $M\left(\Omega_{c c}\right)$ could help distinguish between the two pictures compared here. We also quote the predicted value of $M\left(\Xi_{c c}\right)=3640 \pm$ $12 \mathrm{MeV}$ in the scheme with universal quark masses. This is not as close to the experimental value as that in Ref. [2], but still acceptable. For the $\Omega_{c c}^{*}\left(J^{P}=3 / 2^{+}\right)$we replace the term $-4 a^{\prime} / m_{s} m_{c}=-37.6 \mathrm{MeV}$ in Table III by $+2 a^{\prime} / m_{s} m_{c}=+18.8 \mathrm{MeV}$, so we predict $M\left(\Omega_{c c}^{*}\right)-$ $M\left(\Omega_{c c}\right)=56.4 \mathrm{MeV}$, or $M\left(\Omega_{c c}^{*}\right)=3789 \pm 16 \mathrm{MeV}$.

In addition to the $(c c) s$ binding energy, Table IV contains also the $(b c) s$ and $(b b) s$ binding energies, obtained in an analogous way. The latter are used in Sec. VI to predict the masses $\Omega_{b c}$ and $\Omega_{b b}$.

\section{PRODUCTION AND DECAY}

We can estimate the rate for production of $\Omega_{c c}=c c s$ by reference to that for $\Xi_{c c}^{++}=c c u$. Imagine that some process gives rise to the $(c c)$ diquark, which then fragments into $\Xi_{c c}$ by picking up a $u$ quark. The corresponding process giving rise to $\Omega_{c c}$ then involves $(c c)$ picking up a $s$ quark. What is the ratio of these two processes?

There is information on $b$ quark fragmentation in hadronic collisions from the CDF Collaboration [15], which measures $f_{s} \simeq 0.3 f_{u}$ in $\bar{p} p$ collisions at $\sqrt{s}=$ $1.96 \mathrm{TeV}$. One could expect a similar ratio for $(c c)$ to pick up a $u$ or $s$ quark. At $13 \mathrm{TeV}$, in a sample of $p p$ collisions consisting of an integrated luminosity of $1.7 \mathrm{fb}^{-1}$, the LHCb experiment accumulated $313 \pm 33$ $\Xi_{c c}^{++}$events [1]. One might then expect the same sample to contain about $(100 \pm 10) R \Omega_{c c}^{+}$identifiable events, where $R$ is the ratio of $\Omega_{c c}$ to $\Xi_{c c}$ decays into identifiable branching fractions.

The $\Xi_{c c}$ was seen in the final state $\Lambda_{c} K^{-} \pi^{+} \pi^{+}$. One decay process depicted in Fig. 1 of Ref. [1] involves the initial $u$ and one of the initial charmed quarks $c$ in the $\Xi_{c c}=c c u$ ending up in the $\Lambda_{c}=u c d$. If the initial baryon is $\Omega_{c c}=c c s$, an initial $s$ and one of the initial charmed quarks will end up instead in a $\Xi_{c}^{0}=s c d$. The detectability of the $\Omega_{c c}$ will then depend on the relative efficiencies for reconstruction of $\Xi_{c}^{0}$ and $\Lambda_{c}$.

Another potentially useful decay mode of $\Xi_{c c}^{++}$is into $\pi^{+} \Xi_{c}^{+}$. Its visibility at $\mathrm{LHCb}$ will depend on relative

TABLE V. Summary of predictions of $\Omega_{Q Q}$ masses, in MeV. "Separate" denotes separate quark masses for mesons and baryons; "universal" denotes universal quark masses for mesons and baryons.

\begin{tabular}{lrr}
\hline \hline & Separate & Universal \\
\hline$M\left(\Omega_{c c}\right)$ & $3692 \pm 16$ & $3732 \pm 14$ \\
$M\left(\Omega_{b c}\right)$ & $6968 \pm 19$ & $7013 \pm 16$ \\
$M\left(\Omega_{b c}^{\prime}\right)$ & $6984 \pm 19$ & $7025 \pm 16$ \\
$M\left(\Omega_{b b}\right)$ & $10208 \pm 18$ & $10255 \pm 16$ \\
\hline \hline
\end{tabular}


TABLE VI. Comparison of nonlattice predictions for $M\left(\Omega_{c c}\right)$.

\begin{tabular}{lcl}
\hline \hline & Value & \\
Reference & $(\mathrm{MeV})$ & \\
\hline Present & $3692 \pm 16$ & Separate baryonic quark masses \\
work & & \\
Present & $3732 \pm 14$ & Universal quark masses \\
work & & \\
{$[7]$} & $3730-3940$ & QCD-motivated quark model \\
{$[17]$} & 3690 & Bag model \\
{$[18]$} & 3664 & Bag model \\
{$[19]$} & $3819 \pm 57$ & QCD-motivated quark model \\
{$[20]$} & 3811 & QCD-motivated quark model \\
{$[21]$} & 3703 & Potential models \\
{$[21]$} & 3657 & Bag models \\
{$[22]$} & $3760.7 \pm 2.4^{\text {a }}$ & Potential approach \\
{$[23]$} & 3720 & Potential model \\
{$[24]$} & 3710 & Heavy quark effective theory \\
{$[25]$} & 3737 & Potential model \\
{$[26]$} & $3740 \pm 80$ & Feynman-Hellmann+ semiempirical \\
{$[27]$} & 3787 & Mass sum rules \\
{$[28]$} & 3760 & Relativistic quasipotential quark model \\
{$[29]$} & 3710 & Three-body Faddeev equations \\
{$[30]$} & $3804 \pm 8$ & Quadratic mass relations \\
{$[31]$} & 3598 & Bootstrap quark model +Faddeev eqs. \\
{$[32]$} & $3650 \pm 50$ & Nonrelativistic QCD sum rules \\
{$[33]$} & $3749 \pm 10$ & Quark model \\
{$[34]$} & $3590 \pm 50$ & Potential approach +QCD sum rules \\
{$[35]$} & 3594 & Potential model \\
{$[36]$} & 3860 & Nonperturbative string \\
{$[37]$} & 3778 & Relativistic quark-diquark \\
{$[38]$} & 3619 & Bag model \\
{$[39]$} & $3637 \pm 23$ & Lattice; exact chiral symmetry \\
{$[40]$} & 3732 & Relativistic quark model + Bethe-Salpeter \\
{$[41]$} & $3702+41$ & Variational \\
{$[42]$} & 3815 & Quark model \\
{$[43]$} & 3719 & Relativistic quark model \\
{$[44]$} & $3650.4 \pm 6.3^{b}$ & Quadratic mass relations \\
{$[45]$} & 3697 & Quark model+QCD \\
{$[46]$} & $3710 \pm 140$ & QCD sum rules \\
{$[47]$} & $3635 \pm 15$ & Instantaneous approx + Bethe-Salpeter \\
{$[48]$} & $3566 \div 3687$ & Potential model \\
{$[49]$} & $4250 \pm 200$ & QCD sum rules \\
{$[50]$} & 3710 & Modified bag model \\
{$[51]$} & 3648 & Anti-de Sitter/QCD inspired potl. \\
{$[52]$} & $3630 \mathrm{~b}$ & QCD sum rules \\
{$[53]$} & 3667 & Preferred potential model \\
{$[54]$} & $3650 \pm 40^{\mathrm{b}}$ & Quadratic mass relations \\
\hline \hline
\end{tabular}

${ }^{\mathrm{a}}$ Spin-weighted average of $M\left(\Omega_{c c}\right)$ and $M\left(\Omega_{c c}^{*}\right)$.

${ }^{\mathrm{b}} \mathrm{SELEX}$ [4] $M\left(c c d, 1 / 2^{+}\right)=3519 \mathrm{MeV}$ candidate as input. efficiencies for reconstruction of $\Xi_{c}^{+}$and $\Lambda_{c}$. The corresponding decay mode of $\Omega_{c c}$ is into $\pi^{+} \Omega_{c}$. The LHCb experiment has detected not only the $\Omega_{c}$ but several excited states of it [16] in the final state $\Xi_{c}^{+} K^{-}$, providing a test of ability to reconstruct $\Xi_{c}^{+}$.

\section{MASSES OF $\Omega_{b c}=b c s$ AND $\Omega_{b b}=b b s$}

We have seen that much of the uncertainty in prediction of $M\left(\Omega_{c c}\right)$ lies in uncertainty of the binding energy between the $(c c)$ diquark and the strange quark. The same is true when predicting the masses of $\Omega_{b c}$ and $\Omega_{b b}$. Extrapolating our results for nonstrange states [2] to ones in which $q=u, d$ is replaced with $s$, we take account of (1) the $s-q$ mass difference, (2) differences in $\left(\left(Q Q^{\prime}\right) q\right)$ and $\left(\left(Q Q^{\prime}\right) s\right)$ binding, and (3) small differences in hyperfine splittings, to obtain the results in Table V. The use of universal quark masses raises the prediction of all $\Omega_{Q_{1} Q_{2}}$ masses by about $40 \mathrm{MeV}$.

\section{RESULTS}

Using the same methods used to obtain an accurate prediction of the mass of the recently discovered doublycharmed baryons $\Xi_{c c}^{++}$, we predict the mass of its strange partner: $M\left(\Omega_{c c}\right)=3692 \pm 16 \mathrm{MeV}$. The hyperfine partner of this state, with $J^{P}=3 / 2^{+}$, is predicted to have a mass $M\left(\Omega_{c c}^{*}\right)=3756 \pm 16 \mathrm{MeV}$. Predictions for the ground state masses of the bcs baryons $\Omega_{b c}$ and $\Omega_{b c}^{\prime}$ and the $b b s$ baryon $\Omega_{b b}$ are also presented. The use of universal quark masses with an added "string-junction" contribution for baryons raises these predictions by about $40 \mathrm{MeV}$.

Our predictions for $M\left(\Omega_{c c}\right)$ are compared with a number of others in Tables VI (non-lattice) and VII (lattice). The predictions based on lattice gauge theory are shown separately as they have less of a spread. The corresponding values are plotted in Figs. 1 and 2.

In the picture with separate quark masses for mesons and baryons, the prediction of a rather large value of $B((c c) s)$ distinguishes our approach from a number of others [3] in

TABLE VII. Comparison of lattice predictions for $M\left(\Omega_{c c}\right)$ with our result.

\begin{tabular}{lll}
\hline \hline Reference & \multicolumn{1}{c}{ Value $(\mathrm{MeV})$} & \multicolumn{1}{c}{ Method } \\
\hline Present work & $3692 \pm 16$ & Separate baryonic quark masses \\
Present work & $3732 \pm 14$ & Universal quark masses \\
{$[55]$} & $3747(9)\left(\begin{array}{c}11 \\
47\end{array}\right) \div 3727(9)\left(\begin{array}{c}16 \\
40\end{array}\right)$ & Quenched lattice $(\mathrm{LGT})$ \\
{$[56]$} & $3663(11)(17)(95)$ & Quenched lattice \\
{$[57]$} & $37636 \pm 19 \pm 2_{-79}^{+13}$ & Lattice, domain-wall $+\mathrm{KS}$ fermions \\
{$[58]$} & $3704(5)(16)$ & Lattice, $N_{f}=2+1$ \\
{$[59]$} & $3679(40)(17)(5)$ & LGT, $N_{f}=2+1, m_{\pi}=200 \mathrm{MeV}$ \\
{$[60]$} & $3658(11)(16)(50)$ & LGT, $N_{f}=2+1, m_{\pi}=210 \mathrm{MeV}$ \\
{$[61]$} & $3738(20)(20)$ & Lattice \\
{$[62]$} & $(3640 \pm 173) \div(3663 \pm 230)$ & Lattice; on-shell renormalization \\
{$[63]$} & $3711(5)(30)$ & LGT, clover-improved, physical $m_{\pi}$ \\
\hline \hline
\end{tabular}




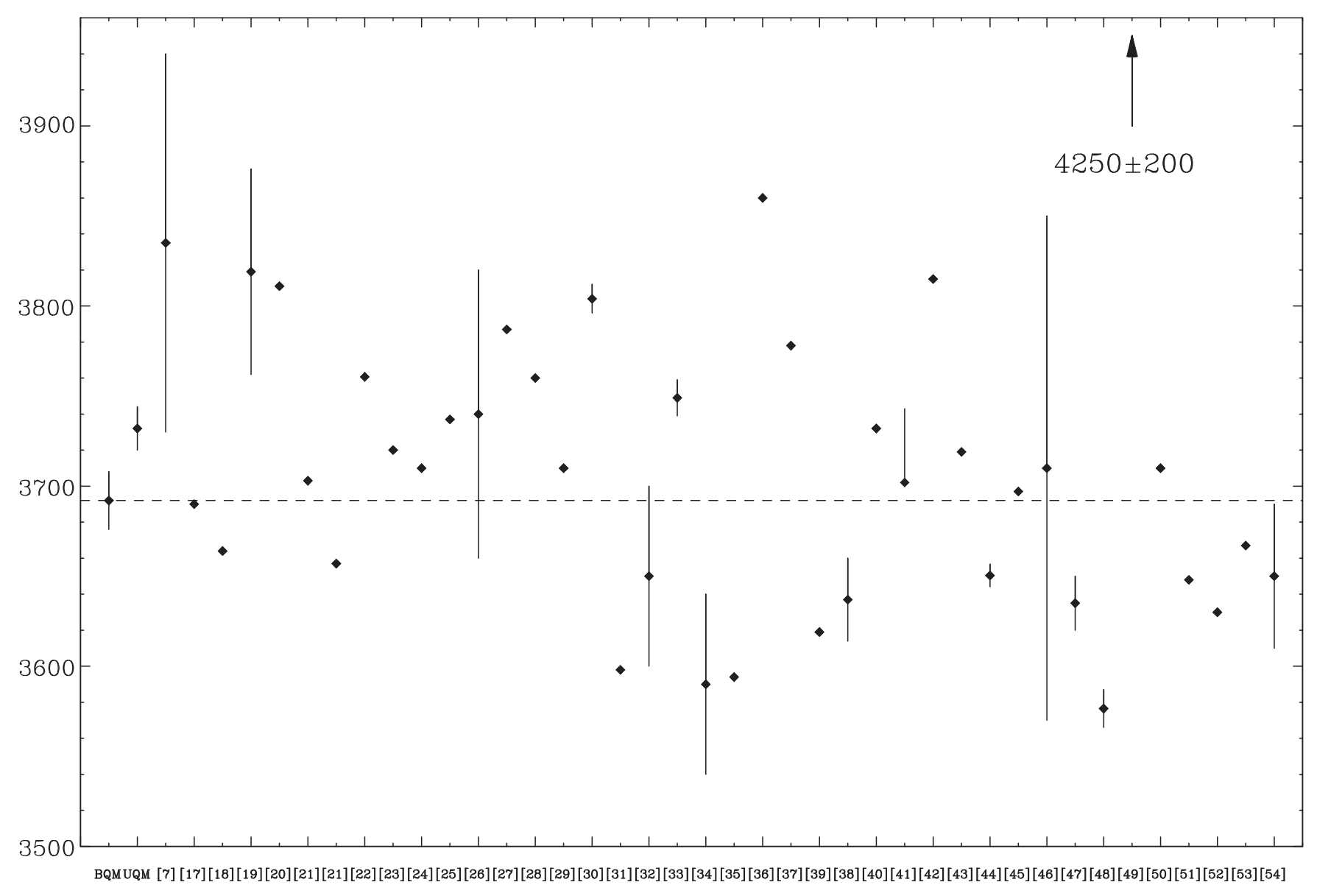

FIG. 1. Comparison of non-lattice predictions for $M\left(\Omega_{c c}\right)$. The first two points are our predictions for baryonic quark mass (BQM; dashed line) and universal quark masses (UQM).

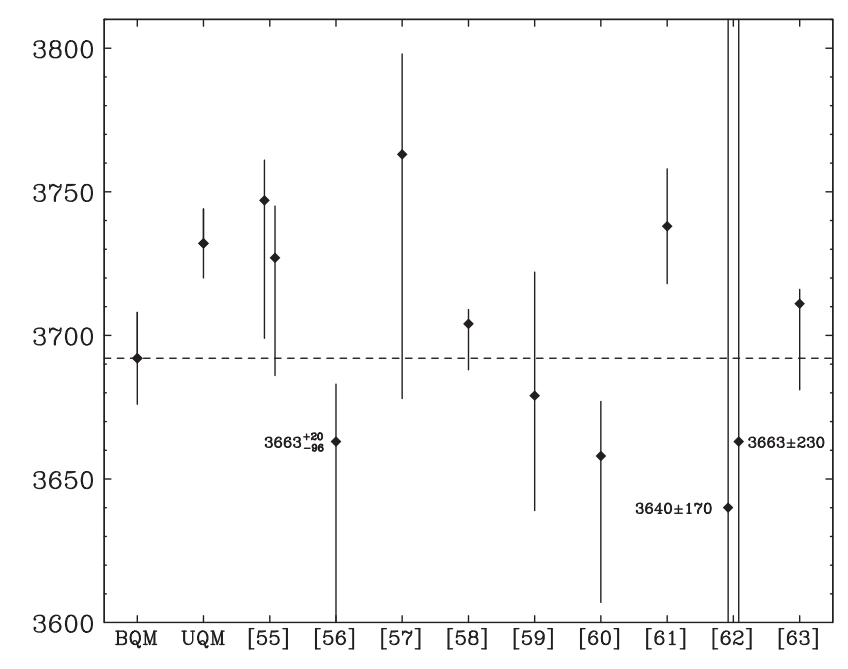

FIG. 2. Comparison of lattice predictions for $M\left(\Omega_{c c}\right)$. The first two points are our (nonlattice) predictions for baryonic quark mass (BQM; dashed line) and universal quark masses (UQM). which the difference $M\left(\Omega_{c c}\right)-M\left(\Xi_{c c}\right)$ is larger than our central value of $65 \mathrm{MeV}$. In our calculation more than half of the mass quark difference $m_{s}^{b}-m_{q}^{b}=175 \mathrm{MeV}$ is canceled by increased binding. For comparison, a lattice gauge theory calculation [61] finds $M\left(\Xi_{c c}\right)=3610(23) \times$ (22) $\mathrm{MeV}, \quad M\left(\Xi_{c c}^{*}\right)=3692(28)(21) \mathrm{MeV}, \quad M\left(\Omega_{c c}\right)=$ $3738(20)(20) \mathrm{MeV}, M\left(\Omega_{c c}^{*}\right)=3820(20)(22) \mathrm{MeV}$, implying a difference between the strange and nonstrange states of $128 \mathrm{MeV}$. This is closer to the value of $105 \mathrm{MeV}$ we find in the picture with universal quark masses.

The production cross section for $\Omega_{c c}$ was estimated to be about 0.3 times that for $\Xi_{c c}^{++}$. Its detectability then depends on the relative efficiency for reconstructing $\Xi_{c}^{0}$ and $\Lambda_{c}$.

\section{ACKNOWLEDGMENTS}

We thank our $\mathrm{LHCb}$ colleagues for encouragement. J. L. R. thanks Tel Aviv University for hospitality during completion of this work. 
[1] R. Aaij et al. (LHCb Collaboration), Observation of the Doubly Charmed Baryon $\Xi_{c c}^{++}$, Phys. Rev. Lett. 119, 112001 (2017).

[2] M. Karliner and J. L. Rosner, Baryons with two heavy quarks: Masses, production, decays, and detection, Phys. Rev. D 90, 094007 (2014).

[3] We refer the reader to Refs. [1,2] for an extensive list of other predictions.

[4] M. Mattson et al. (SELEX Collaboration), First Observation of the Doubly Charmed Baryon $\Xi_{c c}^{++}$, Phys. Rev. Lett. 89, 112001 (2002); A. Ocherashvili et al. (SELEX Collaboration), Confirmation of the double charm baryon $\Xi_{c c}^{++}(3520)$ via its decay to $p D^{+} K^{-}$, Phys. Lett. B 628, 18 (2005); J. Engelfried (SELEX Collaboration), in Heavy quarks and leptons, Proceedings, 8th International Workshop, HQLO6, Munich, Germany (2006), http://www.slac.stanford.edu/ econf/C0610161/.

[5] M. Karliner, B. Keren-Zur, H. J. Lipkin, and J. L. Rosner, The quark model and $b$ baryons, Ann. Phys. (Amsterdam) 324, 2 (2009).

[6] M. Karliner and J. L. Rosner, Isospin splittings in baryons with two heavy quarks, Phys. Rev. D 96, 033004 (2017).

[7] A. De Rujula, H. Georgi, and S. L. Glashow, Hadron masses in a gauge theory, Phys. Rev. D 12, 147 (1975).

[8] H. Hellmann, Acta Physicochim. URSS I 6, 913 (1935); IV 2, 225 (1936); Einführung in die Quantenchemie (F. Deuticke, Leipzig and Vienna, 1937), p. 286; R. P. Feynman, Forces in Molecules, Phys. Rev. 56, 340 (1939).

[9] C. Quigg and J.L. Rosner, Quarkonium level spacings, Phys. Lett. 71B, 153 (1977).

[10] C. Quigg and J.L. Rosner, Quantum mechanics with applications to quarkonium, Phys. Rep. 56, 167 (1979).

[11] H. J. Lipkin, A unified description of mesons, baryons and baryonium, Phys. Lett. B 74, 399 (1978).

[12] S. Gasiorowicz and J. L. Rosner, Hadron spectra and quarks, Am. J. Phys. 49, 954 (1981).

[13] M. Karliner, S. Nussinov, and J. L. Rosner, $Q Q \bar{Q} \bar{Q}$ states: Masses, production, and decays, Phys. Rev. D 95, 034011 (2017).

[14] G. Rossi and G. Veneziano, The string-junction picture of multiquark states: an update, J. High Energy Phys. 06 (2016) 041.

[15] T. Aaltonen et al. (CDF Collaboration), Measurement of ratios of fragmentation fractions for bottom hadrons in $p \bar{p}$ collisions at $\sqrt{s}=1.96 \mathrm{TeV}$, Phys. Rev. D 77, 072003 (2008).

[16] R. Aaij et al. (LHCb Collaboration), Observation of Five New Narrow $\Omega_{c}^{0}$ States Decaying to $\Xi_{c}^{+} K^{-}$, Phys. Rev. Lett. 118, 182001 (2017).

[17] R. L. Jaffe and J.E. Kiskis, Spectra of new hadrons, Phys. Rev. D 13, 1355 (1976).

[18] W. Ponce, Heavy quarks in a spherical bag, Phys. Rev. D 19, 2197 (1979).

[19] J. Bjorken, Report No. FERMILAB-FN-0986-T (unpublished), available online at http://inspirehep.net/record/ 1316585/files/fermilab-fn-0986-t.pdf.

[20] K. Anikeev, D. Atwood, F. Azfar, S. Bailey, C. W. Bauer, W. Bell, G. Bodwin and E. Braaten et al., Workshop on B Physics at Conferences C99-09-23.2 and C00-02-24, arXiv: hep-ph/0201071.
[21] S. Fleck and J. M. Richard, Baryons with double charm, Prog. Theor. Phys. 82, 760 (1989).

[22] B. O. Kerbikov, M. I. Polikarpov, and L. V. Shevchenko, Multi-quark masses and wave functions through modified Green's function Monte Carlo method, Nucl. Phys. B331, 19 (1990).

[23] J. M. Richard, Hadrons with two heavy quarks, eConf No. C94-06-07.1, 95 (1994), https://arxiv.org/abs/hep-ph/ 9407224; E. Bagan, H. G. Dosch, P. Gosdzinsky, S. Narison, and J.-M. Richard, Hadrons with charm and beauty, Z. Phys. C 64, 57 (1994).

[24] J. G. Korner, M. Kramer, and D. Pirjol, Heavy baryons, Prog. Part. Nucl. Phys. 33, 787 (1994).

[25] A. Martin and J. M. Richard, Omega(c) and other charmed baryons revisited, Phys. Lett. B 355, 345 (1995).

[26] R. Roncaglia, D. B. Lichtenberg, and E. Predazzi, Predicting the masses of baryons containing one or two heavy quarks, Phys. Rev. D 52, 1722 (1995).

[27] D. B. Lichtenberg, R. Roncaglia, and E. Predazzi, Mass sum rules for singly and doubly heavy flavored hadrons, Phys. Rev. D 53, 6678 (1996).

[28] D. Ebert, R. N. Faustov, V. O. Galkin, A. P. Martynenko, and V. A. Saleev, Heavy baryons in the relativistic quark model, Z. Phys. C 76, 111 (1997).

[29] B. Silvestre-Brac, Spectroscopy of baryons containing heavy quarks, Prog. Part. Nucl. Phys. 36, 263 (1996).

[30] L. Burakovsky, J. T. Goldman, and L. P. Horwitz, New quadratic baryon mass relations, Phys. Rev. D 56, 7124 (1997).

[31] S. M. Gerasyuta and D. V. Ivanov, Charmed baryons in bootstrap quark model, Nuovo Cimento Soc. Ital. Fis. 112A, 261 (1999).

[32] V. V. Kiselev and A. E. Kovalsky, Doubly heavy baryons Omega $\left(Q Q^{\prime}\right)$ versus $\mathrm{Xi}\left(Q Q^{\prime}\right)$ in sum rules of NRQCD, Phys. Rev. D 64, 014002 (2001).

[33] C. Itoh, T. Minamikawa, K. Miura, and T. Watanabe, Doubly charmed baryon masses and quark wave functions in baryons, Phys. Rev. D 61, 057502 (2000).

[34] V. V. Kiselev and A. K. Likhoded, Baryons with two heavy quarks, Usp. Fiz. Nauk 172, 497 (2002) [Phys. Usp. 45, 455 (2002)].

[35] V. V. Kiselev, A. K. Likhoded, O. N. Pakhomova, and V. A. Saleev, Mass spectra of doubly heavy Omega $Q Q^{\prime}$ baryons, Phys. Rev. D 66, 034030 (2002).

[36] I. M. Narodetskii and M. A. Trusov, in Proceedings of the 9th International Conference on the Structure of Baryons, Jefferson Lab, Newport News, VA (World Scientific, Singapore, 2002); The doubly heavy baryons in the nonperturbative QCD approach, arXiv:hep-ph/0204320.

[37] D. Ebert, R. N. Faustov, V. O. Galkin, and A. P. Martynenko, Mass spectra of doubly heavy baryons in the relativistic quark model, Phys. Rev. D 66, 014008 (2002).

[38] D. H. He, K. Qian, Y. B. Ding, X. Q. Li, and P. N. Shen, Evaluation of spectra of baryons containing two heavy quarks in bag model, Phys. Rev. D 70, 094004 (2004).

[39] , Baryon masses in lattice QCD with exact chiral symmetry, Nucl. Phys. A755, 471 (2005).

[40] S. Migura, D. Merten, B. Metsch, and H.-R. Petry, Charmed baryons in a relativistic quark model, Eur. Phys. J. A 28, 41 (2006). 
[41] C. Albertus, E. Hernandez, J. Nieves, and J. M. VerdeVelasco, Static properties and semileptonic decays of doubly heavy baryons in a nonrelativistic quark model, Eur. Phys. J. A 32, 183 (2007); Erratum, 36, 119(E) (2008).

[42] W. Roberts and M. Pervin, Heavy baryons in a quark model, Int. J. Mod. Phys. A 23, 2817 (2008).

[43] A. P. Martynenko, Ground-state triply and doubly heavy baryons in a relativistic three-quark model, Phys. Lett. B 663, 317 (2008).

[44] X. H. Guo, K. W. Wei, and X. H. Wu, Some mass relations for mesons and baryons in Regge phenomenology, Phys. Rev. D 78, 056005 (2008).

[45] A. Valcarce, H. Garcilazo, and J. Vijande, Towards an understanding of heavy baryon spectroscopy, Eur. Phys. J. A 37, 217 (2008).

[46] Z. G. Wang, Analysis of the $\frac{1}{2}^{+}$doubly heavy baryon states with QCD sum rules, Eur. Phys. J. A 45, 267 (2010).

[47] M.-H. Weng, X.-H. Guo, and A. W. Thomas, Bethe-Salpeter equation for doubly heavy baryons in the covariant instantaneous approximation, Phys. Rev. D 83, 056006 (2011).

[48] B. Patel, A. K. Rai, and P. C. Vinodkumar, Heavy flavour baryons in hyper central model, Pramana 70, 797 (2008).

[49] J. R. Zhang and M. Q. Huang, Doubly heavy baryons in QCD sum rules, Phys. Rev. D 78, 094007 (2008).

[50] A. Bernotas and V. Simonis, Heavy hadron spectroscopy and the bag model, Lith. J. Phys. 49, 19 (2009).

[51] F. Giannuzzi, Doubly heavy baryons in a Salpeter model with AdS/QCD inspired potential, Phys. Rev. D 79, 094002 (2009).

[52] L. Tang, X. H. Yuan, C. F. Qiao, and X. Q. Li, Study of doubly heavy baryon spectrum via QCD sum rules, Commun. Theor. Phys. 57, 435 (2012).
[53] Z. Ghalenovi, A. A. Rajabi, S. x. Qin, and D. H. Rischke, Ground-state masses and magnetic moments of heavy baryons, Mod. Phys. Lett. A 29, 1450106 (2014).

[54] K. W. Wei, B. Chen, and X. H. Guo, Masses of doubly and triply charmed baryons, Phys. Rev. D 92, 076008 (2015).

[55] R. Lewis, N. Mathur, and R. M. Woloshyn, Charmed baryons in lattice QCD, Phys. Rev. D 64, 094509 (2001).

[56] J. M. Flynn, F. Mescia, and A. Shams Bin Tariq (UKQCD Collaboration), Spectroscopy of doubly charmed baryons in lattice QCD, J. High Energy Phys. 07 (2003) 066.

[57] L. Liu, H.-W. Lin, K. Orginos, and A. Walker-Loud, Singly and doubly charmed $\mathrm{J}=1 / 2$ baryon spectrum from lattice QCD, Phys. Rev. D 81, 094505 (2010).

[58] Y. Namekawa et al. (PACS-CS Collaboration), Charmed baryons at the physical point in $2+1$ flavor lattice QCD, Phys. Rev. D 87, 094512 (2013).

[59] R. A. Briceno, H. W. Lin, and D. R. Bolton, Charmedbaryon spectroscopy from lattice QCD with $N_{f}=2+$ $1+1$ flavors, Phys. Rev. D 86, 094504 (2012).

[60] C. Alexandrou, V. Drach, K. Jansen, C. Kallidonis, and G. Koutsou, Baryon spectrum with $N_{f}=2+1+1$ twisted mass fermions, Phys. Rev. D 90, 074501 (2014).

[61] Z. S. Brown, W. Detmold, S. Meinel, and K. Orginos, Charmed bottom baryon spectroscopy from lattice QCD, Phys. Rev. D 90, 094507 (2014).

[62] Z.F. Sun and M. J. Vicente Vacas, Masses of doubly charmed baryons in the extended on-mass-shell renormalization scheme, Phys. Rev. D 93, 094002 (2016).

[63] C. Alexandrou and C. Kallidonis, Low-lying baryon masses using $N_{f}=2$ twisted mass clover-improved fermions directly at the physical point, Phys. Rev. D 96, 034511 (2017). 